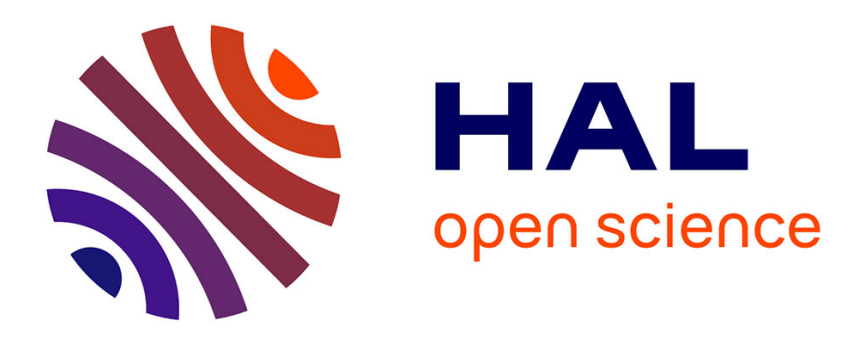

\title{
Antenna Selection for Array Synthesis Problems
}

Benjamin Fuchs

\section{To cite this version:}

Benjamin Fuchs. Antenna Selection for Array Synthesis Problems. IEEE Antennas and Wireless Propagation Letters, 2017, 16, pp.868-871. 10.1109/LAWP.2016.2612762 . hal-01534211

\section{HAL Id: hal-01534211 https://hal-univ-rennes1.archives-ouvertes.fr/hal-01534211}

Submitted on 21 Jan 2021

HAL is a multi-disciplinary open access archive for the deposit and dissemination of scientific research documents, whether they are published or not. The documents may come from teaching and research institutions in France or abroad, or from public or private research centers.
L'archive ouverte pluridisciplinaire HAL, est destinée au dépôt et à la diffusion de documents scientifiques de niveau recherche, publiés ou non, émanant des établissements d'enseignement et de recherche français ou étrangers, des laboratoires publics ou privés. 


\title{
Antenna Selection for Array Synthesis Problems
}

\author{
Benjamin Fuchs, Senior Member, IEEE
}

\begin{abstract}
The problem of choosing a set of $N$ antennas from $M$ possible radiators to optimize the performances of antenna arrays is addressed. Solving this combinatorial problem by evaluating all possible choices is untractable unless both $N$ and $M$ are small. Convex relaxations are proposed to solve approximately this antenna selection problem and quickly get bounds on the performance that can be achieved over all possible antenna combinations. Two approaches are also presented to rapidly construct, from these approximated results, good suboptimal solutions, i.e. good antenna selections. For $(N, K)=$ $(30,10)$, i.e. $10^{30}$ possible antenna arrays, the methods can be carried out in a few seconds on a laptop. The proposed approaches are applied to solve various representative numerical instances, they provide quickly valuable information for antenna array designers.
\end{abstract}

Index Terms-Antenna selection, array antennas, semidefinite programming.

\section{INTRODUCTION}

$\mathbf{T}$ HE selection of the best combination of antennas to optimize the performances of an array is a problem that arises in many practical applications. The antenna selection problem has been addressed in MIMO systems [1], sparse arrays design [2] and multicast beamforming problems [3].

This combinatorial problem is also of uppermost importance for array synthesis problems where the choice among a given set of available radiators to form an array is a frequently encountered issue. The selection of quantized array excitations, antenna types, and antenna's locations in order to minimize the sidelobes of a focused beampattern are examples of problems considered in this paper. To formalize the selection problem, let us consider an array composed of $N$ antennas. The objective is to select for each of these $N$ antennas, one element among $K$ possibilities, as represented in Fig. 1, in order to optimize the array radiation performances. Solving this antenna selection problem by evaluating the $K^{N}$ possible array combinations is untractable unless both $K$ and $N$ are very small. As an example, the selection of $N=30$ antennas with $K=10$ possibilities leads to $10^{30}$ possible choices. A direct enumeration is clearly not possible. Such combinatorial problems can be optimally solved using global optimization techniques such as the branch and bound algorithm (denoted BB) [4], [5]. However, in the worst case, these algorithms require an effort that grows exponentially with problem size but they can be used as a reference for problems with modest values of $(N, K)$. Evolutionary techniques, such as binary genetic algorithm [6] or particle swarm optimization [7], can also be applied to solve mixed integer problems and consequently combinatorial problems but without any guarantee of

Manuscript received xx, 2016; revised xx, xx

The author is with the IETR / University of Rennes I, France. (e-mail: benjamin.fuchs@univ-rennes1.fr) convergence towards the optimal solution.

In this paper, we propose two approaches for approximately solving antenna selection problems. The originally combinatorial optimization problem is relaxed to be rewritten as a semidefinite program [8]. The so-approximated problem is then convex and therefore efficiently solvable. For $(N, K)=$ $(30,10)$, the methods can be carried out in a few seconds on a $2.8 \mathrm{GHz}$ personal computer. They provide quickly both a suboptimal selection of antennas and a bound on the performances that can be achieved by any selection of antennas. Although there is no guarantee that the gap between the performance of the selected antenna array and the optimal one is always small, numerical experiments suggest that relevant bounds are obtained in various representative cases. The idea of using convex relaxation as the basis for a heuristic for solving a combinatorial problem is not new [8]-[10]. Our goal is to show that such approaches are also of interest for antenna selection problems.

The paper is organized as follows. The antenna selection problem is described and formalized in Section II. Two convex relaxation approaches are proposed and explained in Section III to approximately solve the combinatorial problem and get bounds on the best achievable performance. A simple heuristic and an algorithm to derive sub-optimal solutions are detailed in Section IV. Numerical examples are provided in Section $\mathrm{V}$ to illustrate the interest of the proposed approaches and conclusions are drawn in Section VI.

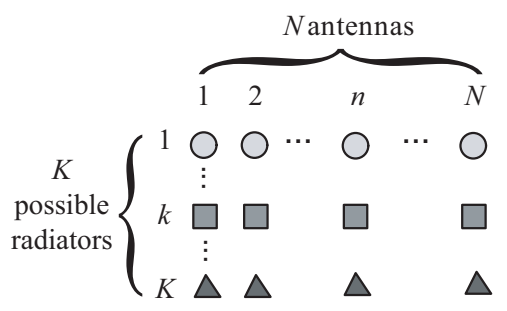

Fig. 1. Antenna selection problem: choice of one among $K$ potential radiators to form an array composed of $N$ antennas.

\section{Problem Formulation}

The goal of the antenna selection problem is to choose for each of the $N$ antennas of the array, one element among $K$ potential radiators to optimize the performances of the array. To formalize the problem, we introduce the vector $\boldsymbol{x}$ of dimension $M=K \times N$ that is a concatenation of $N$ subvectors $\boldsymbol{x}_{n}$ of dimension $K$ :

$$
\begin{aligned}
& \boldsymbol{x}=\left[\begin{array}{lllll}
\boldsymbol{x}_{1}{ }^{T}, & \ldots & \boldsymbol{x}_{n}{ }^{T}, & \ldots & , \boldsymbol{x}_{N}{ }^{T}
\end{array}\right]^{T} \\
& \text { with } \boldsymbol{x}_{n}{ }^{T}=\left[\begin{array}{lllll}
x_{n, 1} & \ldots & x_{n, k} & \ldots & x_{n, K}
\end{array}\right]
\end{aligned}
$$


where the boolean $x_{n, k} \in\{0,1\}$ encodes whether the antenna $(n, k)$ is to be selected. The subvector $\boldsymbol{x}_{n}$ corresponds to the choice for the antenna $n$. Only one antenna is chosen which means that $\mathbf{1}^{T} \boldsymbol{x}_{n}=1$ for $n=1, \ldots, N$, where $\mathbf{1}$ is a vector of length $K$ with all entries one.

Let us consider the optimization of the radiation performances $\mathcal{P}$ of the array under some constraints $\mathcal{C}$ in order to formulate the antenna selection problem. Both the objective function $\mathcal{P}(\boldsymbol{x})$ and constraints $f(\boldsymbol{x}) \in \mathcal{C}$ are assumed convex. A typical array synthesis requirement is to maximize the power in a given direction (objective $\max _{\boldsymbol{x}} \mathcal{P}(\boldsymbol{x})$ ) while the sidelobes are constrained below a predefined value (constraints $f(\boldsymbol{x}) \in \mathcal{C}$ ). The problem of selecting the best subset of $N$ antennas can then be expressed as the following optimization problem:

$$
\max _{\boldsymbol{x}} \mathcal{P}(\boldsymbol{x}) \quad \text { subject to }\left\{\begin{array}{ll}
f(\boldsymbol{x}) \in \mathcal{C} & \\
\mathbf{1}^{T} \boldsymbol{x}_{n}=1, & \forall n \\
x_{n, k}=\{0,1\}, & \forall(n, k)
\end{array} .\right.
$$

This optimization problem is hard to solve because of the last $N \times K$ constraints that restrict the components of $\boldsymbol{x}_{n}$ to be boolean. We note $\boldsymbol{x}^{*}$ the optimal solution of (2) and $\mathcal{P}^{*}=\mathcal{P}\left(\boldsymbol{x}^{*}\right)$ the best achievable performance.

\section{Approximate Solutions Via Convex RELAXATIONS}

Two convex relaxations of the boolean optimization problem (2) are presented. We denote $\tilde{\boldsymbol{x}}$ the optimal solutions of these approximated problems and $\widetilde{\mathcal{P}}$ the associated objective value. The results obtained by the relaxations provide a cheaply computable bound on the best performance $\mathcal{P}^{*}$ that can be achieved solving the original selection problem (2). For a maximization problem such as $(2)$, it means that $\mathcal{P}^{*} \leq \widetilde{\mathcal{P}}$.

\section{A. Continuous Relaxation (CTS)}

A simple upper bound on $\mathcal{P}^{*}$ can be obtained by relaxing the boolean constraint $x_{n, k} \in\{0,1\}$ of (2) into $0 \leq x_{n, k} \leq$ 1, $\forall(n, k)$. The optimization problem becomes convex and can then be optimally solved efficiently using, for instance, interior point methods. The optimal solution $\widetilde{\boldsymbol{x}}$ can (and will) have components that are real numbers and not boolean. It is therefore not a feasible point of (2). Nevertheless, we have $\mathcal{P}^{*} \leq \widetilde{\mathcal{P}}$ but not much can be said about the tightness, i.e. the difference $\mathcal{P}^{*}-\widetilde{\mathcal{P}}$, of this upper bound.

\section{B. SemiDefinite Relaxation (SDR)}

The boolean constraints $x_{n, k}=\{0,1\}$ are equivalent to:

$$
x_{n, k}\left(x_{n, k}-1\right)=0 \text { or } x_{n, k}^{2}=x_{n, k}, \quad \forall(n, k) .
$$

With the notations introduced in (1), these constraints can be rewritten:

$$
\operatorname{diag}(\boldsymbol{X})=\boldsymbol{x} \text { with } \boldsymbol{X}=\boldsymbol{x} \boldsymbol{x}^{T} .
$$

We can relax (4) by replacing the non convex equality $\boldsymbol{X}=\boldsymbol{x} \boldsymbol{x}^{T}$ with a convex positive semidefiniteness constraint $\boldsymbol{X} \succeq \boldsymbol{x} \boldsymbol{x}^{T}$ that can be formulated as a Schur complement (see appendix A.5.5 of [11]). The antenna selection problem becomes:

$$
\max _{\boldsymbol{x}, \boldsymbol{X}} \mathcal{P}(\boldsymbol{x}) \quad \text { subject to }\left\{\begin{array}{l}
f(\boldsymbol{x}) \in \mathcal{C} \\
\mathbf{1}^{T} \boldsymbol{x}_{n}=1, \forall n \\
0 \leq x_{n, k} \leq 1, \forall(n, k) \\
\operatorname{diag}(\boldsymbol{X})=\boldsymbol{x} \\
{\left[\begin{array}{cr}
\boldsymbol{X} & \boldsymbol{x} \\
\boldsymbol{x}^{T} & 1
\end{array}\right] \succeq 0}
\end{array}\right.
$$

The optimization problem (5) is a semidefinite program, its optimal solution $\left(\boldsymbol{x}^{\text {sdr }}, \boldsymbol{X}^{\text {sdr }}\right)$ can be found efficiently. It can be shown that $\boldsymbol{x}^{\text {sdr }}$ is equal to $\widetilde{\boldsymbol{x}}$ the solution of the continuous and semidefinite relaxation leads to the same performance value $\widetilde{\mathcal{P}}$. Nevertheless, the matrix $\boldsymbol{X}^{\text {sdr }}$ will be useful to find good sub-optimal solutions as described in the next Section.

\section{Sub-Optimal Solutions}

The optimal solution $\widetilde{\boldsymbol{x}}$ of the continuous and semidefinite relaxation have real number components. Therefore, they are not solutions of (2) which means that they do not provide a selection of antennas. Two approaches are proposed to derive from $\tilde{\boldsymbol{x}}$ and $\left(\boldsymbol{x}^{\mathrm{sdr}}, \boldsymbol{X}^{\mathrm{sdr}}\right)$ sub-optimal solutions, i.e. feasible points of (2). The associated objective values are denoted $\mathcal{P}^{\text {cts }}$ and $\mathcal{P}^{\text {sdr }}$ respectively, they are lower bounds of $\mathcal{P}^{*}$ and more specifically $\mathcal{P}^{\text {cts }} \leq \mathcal{P}^{\text {sdr }} \leq \mathcal{P}^{*}$.

\section{A. Simple heuristic}

There are many ways to project an approximate solution $\widetilde{\boldsymbol{x}}$ having real number components into a boolean vector $\boldsymbol{x}$ that is a feasible point of (2). The simplest heuristic is to enforce the largest component of each subvector $\widetilde{\boldsymbol{x}}_{n}$ to be equal to unity, whereas the others are all set to zero (ties can be broken arbitrarily). It means that the components of the boolean vector $\boldsymbol{x}$ are determined as follows:

$$
x_{n, k}=\left\{\begin{array}{ll}
1 & \text { if } x_{n, k}=\max \left(\widetilde{\boldsymbol{x}}_{n}\right) \\
0 & \text { otherwise }
\end{array} \forall(n, k) .\right.
$$

Another strategy consists of keeping only the $K^{\prime}$ largest components of $\widetilde{\boldsymbol{x}}_{n}$ (where $1<K^{\prime}<K$ ) and solve the so-reduced antenna selection problem. This step can be applied iteratively. The objective reached with this sub-optimal solution is denoted $\mathcal{P}^{\text {cts }}$.

\section{B. Randomized Algorithm}

The semidefinite relaxation (5) has a probabilistic interpretation that can be used to obtain good feasible points of (2). If $\left(\boldsymbol{x}^{\mathrm{sdr}}, \boldsymbol{X}^{\mathrm{sdr}}\right)$ is the optimal solution of (5), then $\boldsymbol{X}^{\mathrm{sdr}}-\boldsymbol{x}^{\mathrm{sdr}} \boldsymbol{x}^{\mathrm{sdr}}{ }^{T}$ is a covariance matrix. Now if we choose $z$ as a Gaussian random variable of mean $\boldsymbol{\mu}=\boldsymbol{x}^{\mathrm{sdr}}$ and covariance matrix $\boldsymbol{\Sigma}=\boldsymbol{X}^{\mathrm{sdr}}-\boldsymbol{x}^{\mathrm{sdr}} \boldsymbol{x}^{\mathrm{sdr}}{ }^{T}$, then $\boldsymbol{z}$ will solve the original non convex problem (2) "on average" over this distribution.

It means that $z$ solves:

$$
\max _{z} \mathrm{E}\{\mathcal{P}(\boldsymbol{z})\} \quad \text { subject to }\left\{\begin{array}{l}
\mathrm{E}\{f(\boldsymbol{z})\} \in \mathcal{C} \\
\mathrm{E}\left\{\mathbf{1}^{T} \boldsymbol{z}_{n}\right\}=1, \forall n \\
0 \leq \mathrm{E}\left\{z_{n, k}\right\} \leq 1, \quad \forall(n, k) \\
\mathrm{E}\left\{z_{n, k}^{2}\right\}=\mathrm{E}\left\{z_{n, k}\right\} \forall(n, k)
\end{array}\right.
$$


where $\mathrm{E}\{$.$\} denotes the statistical expectation.$

Sampling $z$ from the distribution $\mathcal{N} \sim(\boldsymbol{\mu}, \boldsymbol{\Sigma})$ does not immediately provide a solution to (2) but a feasible point can easily be derived using (6). Thus, a good feasible point can be obtained by sampling $z$ a sufficient number of times $P$ and keeping the best feasible one. With these observations, we construct the randomized algorithm 1 . The outputs of this algorithm are the antenna selection $\boldsymbol{x}^{\mathrm{sdr}}$ and its associated performance $\mathcal{P}^{\text {sdr }}$.

The total number of iterations $P$ of the algorithm 1 is set to $5 M$ as it has been empirically observed that a higher $P$ does not improve significantly the results.

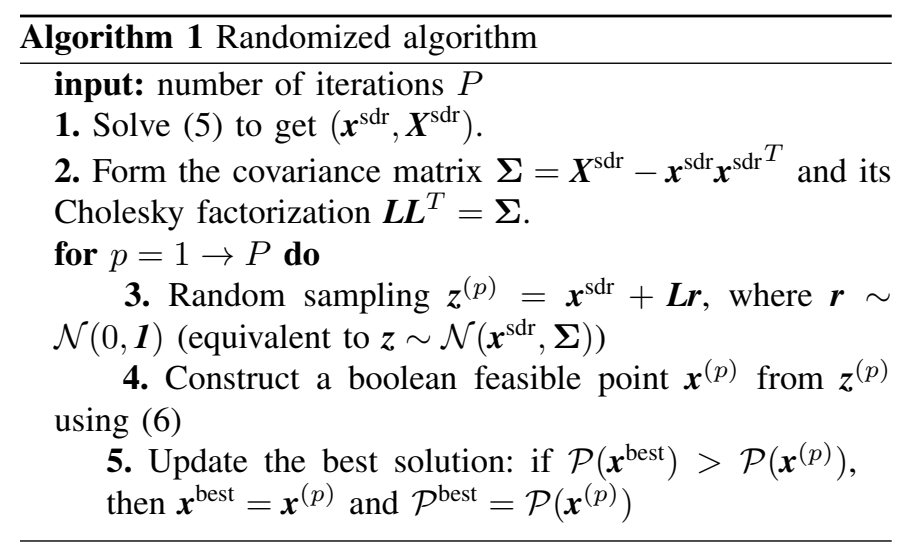

\section{Numerical APPLiCATIONS}

Various antenna selection problems are considered in order to assess the proposed approaches. The goal is to find the best antenna combination (with various quantized excitations, element patterns or locations) in order to maximize the difference (denoted $\mathcal{P}$ ) between main lobe and sidelobe level, problem described in [12]. The optimal performance $\mathcal{P}^{*}$ is computed using a branch and bound algorithm implemented by the optimization software Gurobi [13]. The bounds $\widetilde{\mathcal{P}}, \mathcal{P}^{\text {cts }}$ and $\mathcal{P}^{\text {sdr }}$ are obtained solving the relaxed convex optimization problems using the software CVX, a package for specifying and solving convex programs [14]. All simulations are carried out on a $2.8 \mathrm{GHz}-\mathrm{CPU}$ personal computer and the computation times to get the solution with the branch and bound algorithm, the SDR relaxation and the continuous relaxation are denoted $t^{\mathrm{bb}}, t^{\mathrm{sdr}}$ and $t^{\mathrm{cts}}$, respectively.

\section{A. Excitation Amplitude and Phase Quantification}

We consider a linear array composed of 10 patches working at $10 \mathrm{GHz}$ and simulated with the electromagnetic full wave software Ansys HFSS. For each patch, the choice between $K=20,30$ and 40 complex excitations is proposed. Specifically, the excitations can take the following values: $\left|w_{m}\right|=m / N$ for $m=1, \ldots, 5$ and $\angle w_{n}=n \cdot \frac{2 \pi}{K / 5}$ for $n=0, \ldots, K / 5-1$. The optimization goal is to find the best combination of discrete excitations (among the $10^{20}, 10^{30}$, $10^{40}$ possible choices) in order to minimize the sidelobes for $\theta \in\left[-90^{\circ}, 25^{\circ}\right] \cup\left[55^{\circ}, 90^{\circ}\right]$ of an off-centered focused beampattern (Fig. 2(a)).
TABLE I

Excitation Amplitude and Phase Quantification - CASE of 10 PATCHES ARRAY

\begin{tabular}{ccccc}
\hline \hline$(N, K)$ & $\widetilde{\mathcal{P}}$ & $\mathcal{P}^{*}$ & $\mathcal{P} \mathrm{sdr}$ & $\mathcal{P}^{\mathrm{cts}}$ \\
\hline$(10,20)$ & $19.2 \mathrm{~dB}$ & $10.9 \mathrm{~dB}$ & $10.3 \mathrm{~dB}$ & $6.9 \mathrm{~dB}$ \\
& - & $t^{\mathrm{bb}} / t^{\mathrm{cts}}=122$ & $t^{\mathrm{sdr}} / t^{\mathrm{cts}}=5.1$ & \\
$(10,30)$ & $19.2 \mathrm{~dB}$ & $14.8 \mathrm{~dB}$ & $14.6 \mathrm{~dB}$ & $13.1 \mathrm{~dB}$ \\
& - & $t^{\mathrm{bb}} / t^{\mathrm{cts}}=116$ & $t^{\mathrm{sdr}} / t^{\mathrm{cts}}=6.3$ & \\
$(10,40)$ & $19.2 \mathrm{~dB}$ & $15.9 \mathrm{~dB}$ & $14.4 \mathrm{~dB}$ & $12.5 \mathrm{~dB}$ \\
& - & $t^{\mathrm{bb}} / t^{\mathrm{cts}}=44.4$ & $t^{\mathrm{sdr}} / t^{\mathrm{cts}}=7.7$ & \\
$(10,100)$ & $19.2 \mathrm{~dB}$ & - & $15.1 \mathrm{~dB}$ & $13.3 \mathrm{~dB}$ \\
& - & - & $t^{\mathrm{sdr}} / t^{\mathrm{cts}}=13.6$ & \\
\hline \hline
\end{tabular}

TABLE II

ELEMENT TyPe SELECTION

\begin{tabular}{ccccc}
\hline \hline$(N, K)$ & $\widetilde{\mathcal{P}}$ & $\mathcal{P}^{*}$ & $\mathcal{P}^{\mathrm{sdr}}$ & $\mathcal{P}^{\mathrm{cts}}$ \\
\hline$(10,4)$ & $20.8 \mathrm{~dB}$ & $16.0 \mathrm{~dB}$ & $15.2 \mathrm{~dB}$ & $13.9 \mathrm{~dB}$ \\
& - & $t^{\mathrm{bb}} / t^{\mathrm{cts}}=7.6$ & $t^{\mathrm{sdr}} / t^{\mathrm{cts}}=1.9$ & \\
$(15,4)$ & $33.6 \mathrm{~dB}$ & $20.7 \mathrm{~dB}$ & $19.1 \mathrm{~dB}$ & $13.9 \mathrm{~dB}$ \\
& - & $t^{\mathrm{bb}} / t^{\mathrm{cts}}=18.4$ & $t^{\mathrm{sdr}} / t^{\mathrm{ts}}=2.2$ & \\
$(20,4)$ & $24.2 \mathrm{~dB}$ & $20.6 \mathrm{~dB}$ & $18.0 \mathrm{~dB}$ & $15.4 \mathrm{~dB}$ \\
& - & $t^{\mathrm{bb}} / t^{\mathrm{cts}}=549.6$ & $t^{\mathrm{sdr}} / t^{\mathrm{cts}}=1.5$ & \\
\hline \hline
\end{tabular}

The antenna selection results are reported in Table I. The SDR approach provides a good selection of antenna excitations, since the value $\mathcal{P}^{\text {sdr }}$ is close to the one of reference $\mathcal{P}^{*}$ provided by the branch and bound algorithm. Moreover, this good antenna selection is very fast, $t^{\text {sdr }} / t^{\text {cts }}$ is indeed several orders of magnitude lower than $t^{\mathrm{bb}} / t^{\mathrm{cts}}$. As illustrative example, the case $(N, K)=(10,100)$ is reported to show that the computation time using the SDR approach remains reasonable even for very large combinatorial problems. Note that the branch and bound algorithm cannot be run with our computer from a problem of size larger than $(N, K)=(10,50)$.

\section{B. Type of Antennas}

We now consider a linear array composed of $N$ elements uniformly spaced by $0.7 \lambda$. For each element, the choice between four types of antennas is possible as represented in Fig. 2(b). Classical analytical formulas, that can be found in antenna textbooks such as [15], are used to emulate the Eplane patterns radiated by: a $\lambda / 2$ dipole, an half-wavelength patch, a slot of thickness $\lambda / 4$ on a ground plane and a pyramidal horn of aperture $1 \lambda$. The goal is to find the best combination of antennas in order to minimize the sidelobes for $\theta \in\left[-90^{\circ},-8^{\circ}\right] \cup\left[8^{\circ}, 90^{\circ}\right]$ of a broadside focused beampattern. The results and computation times are reported in Table II. They confirm the previous observations, namely a good selection of antennas is quickly computed using the SDR approach. Here again, the branch and bound algorithm cannot handle a larger combinatorial problem with $(N, K)=(30,4)$ for instance.

\section{Antennas' Location}

We consider a linear array composed of $N$ isotropic elements of length $L=N \lambda / 2$. For each element, $K$ possible 


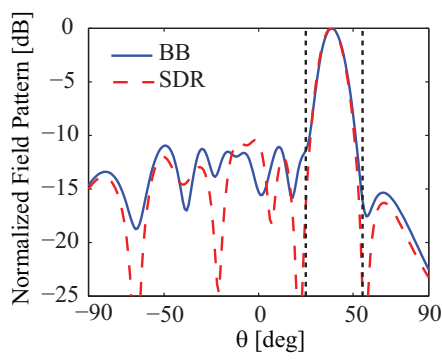

(a)

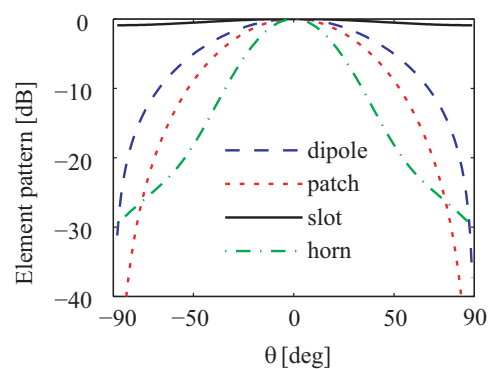

(b)

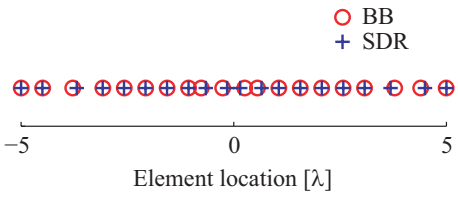

(c)

Fig. 2. (a) Far field patterns radiated by the 10 patches array after the selection between $K=20$ excitation amplitude and phase quantifications. The vertical dotted lines represent the boundaries between main beam and sidelobe region. The achieved performances are: $\mathcal{P}^{*}=10.9 \mathrm{~dB}$ and $\mathcal{P}^{\mathrm{sdr}}=10.3 \mathrm{~dB}$. $(\mathrm{b}) \mathrm{Choice}$ between $K=4$ element patterns corresponding to the E-planes radiation patterns of: a $\lambda / 2$ dipole, a patch of length $\lambda / 2$, a slot of thickness $\lambda / 4$ and a pyramidal horn of aperture $1 \lambda$. (c) Results of the antenna location selection problem with $(N, K)=(20,5)$ : location of the antennas.

TABLE III

ANTENNA Location SELEction

\begin{tabular}{ccccc}
\hline \hline$(N, K)$ & $\widetilde{\mathcal{P}}$ & $\mathcal{P}^{*}$ & $\mathcal{P}^{\mathrm{sdr}}$ & $\mathcal{P}^{\mathrm{cts}}$ \\
\hline$(10,5)$ & $14.2 \mathrm{~dB}$ & $14.1 \mathrm{~dB}$ & $13.3 \mathrm{~dB}$ & $10.9 \mathrm{~dB}$ \\
& - & $t^{\mathrm{bb}} / t^{\mathrm{cts}}=0.75$ & $t^{\mathrm{sdr}} / t^{\mathrm{cts}}=1.75$ & \\
$(20,5)$ & $19.4 \mathrm{~dB}$ & $19.2 \mathrm{~dB}$ & $18.4 \mathrm{~dB}$ & $17.4 \mathrm{~dB}$ \\
& - & $t^{\mathrm{bb}} / t^{\mathrm{cts}}=3.11$ & $t^{\mathrm{sdr}} / t^{\mathrm{cts}}=2.44$ & \\
$(30,5)$ & $27.5 \mathrm{~dB}$ & $26.2 \mathrm{~dB}$ & $23.9 \mathrm{~dB}$ & $18.4 \mathrm{~dB}$ \\
& - & $t^{\mathrm{bb}} / t^{\mathrm{cts}}=415$ & $t^{\mathrm{sdr}} / t^{\mathrm{cts}}=3.33$ & \\
\hline \hline
\end{tabular}

locations are proposed and more specifically $K N$ locations are uniformly spaced over $L$. Note that it is also possible to propose antenna's locations not uniformly spread but around specific positions in order to ensure a minimum distance between elements. The goal is to find the best set of antenna locations in order to minimize the sidelobes for $\sin \theta \in$ $[-1,-0.15] \cup[0.15,1]$ of a broadside focused beampattern. This synthesis problem amounts to design isophoric arrays, i.e. arrays of elements with the same excitations and whose locations are optimized from among a given discrete set in this case. The results of this antenna location selection problem are provided in Table III and the optimized antenna's locations are plotted in Fig. 2(c). Tight upper bounds are obtained via the convex relaxations and good selections of antenna location are also determined with the randomized algorithm in computation times several orders of magnitude faster than the branch and bound algorithm.

\section{CONCLUSION}

The problem of selecting a combination of antennas, from among a set of possible radiators, to optimize the array radiation performances is a difficult combinatorial problem. We have shown that convex relaxation followed by a probabilistic interpretation of the solution enables to quickly both obtain a bound on the best achievable array performance and make a good antenna selection. This general antenna selection formulation encompasses many array synthesis problems of important practical interest. Numerical results on the selection of quantized array excitations, antenna types, and antenna's locations in order to minimize the sidelobes of a focused beampattern have been presented. The proposed approaches do not give a prior guarantee on the tightness of the bound and the goodness of the antenna selection but various realistic numerical experiments show that the antenna selections are often close to the best choices. Therefore, we believe that the proposed approach is useful to rapidly estimate whether given antenna array radiation requirements are achievable or not, and if so to find a good array design.

\section{REFERENCES}

[1] M. Gharavi-Alkhansari and A.B. Gershman, "Fast Antenna Subset Selection in MIMO Systems," IEEE Trans. Signal Processing, vol. 52, no. 2, pp. 339-347, Feb. 2004.

[2] S.E. Nai, W. Ser, Z. Liang Yu and H. Chen, "Beampattern synthesis for linear and planar arrays with antenna selection by convex optimization," IEEE Trans. Antennas Propag., vol. 58, no. 12, pp. 3923-3930, Dec. 2010.

[3] O. Mehanna, N.D. Sidiropoulos, G.B. Giannakis, "Joint Multicast Beamforming and Antenna Selection,” IEEE Trans. Signal Processing, vol. 61, no. 10, pp. 2660-2674, May 2013.

[4] P.M. Narendra and K. Fukunaga, "A branch and bound algorithm for feature subset selection," IEEE Transactions on Computers, vol. 26, no. 9, pp. 917-922, 1977.

[5] S. Boyd and J. Mattingley, "Branch and Bound Methods," March 2007 [Online]. Available: http://web.stanford.edu/class/ee392o/bb.pdf

[6] R.L. Haupt, "Antenna Design With a Mixed Integer Genetic Algorithm," IEEE Trans. Antennas Propag., vol. 55, no. 3, pp. 577-582, Mar. 2007.

[7] N. Jin, Y. Rahmat-Samii, "Hybrid Real-Binary Particle Swarm Optimization (HPSO) in Engineering Electromagnetics," IEEE Trans. Antennas Propag., vol. 58, no. 12, pp. 3786-3794, Dec. 2010.

[8] S. Boyd and L. Vandenberghe, "Semidefinite Programming Relaxations of Non-Convex Problems in Control and Combinatorial Optimization," Communications, Computation, Control and Signal Processing: A Tribute to Thomas Kailath, editor: Kluwer, Chapter 15, pp. 279-288, 1997.

[9] A. d'Aspremont and S. Boyd, "Relaxations and Randomized Methods for Nonconvex QCQPs," fall 2003 [Online]. Available: http://www.stanford.edu/class/ee392o/relaxations.pdf

[10] Z-Q. Luo and T.-H. Chang, "SDP relaxation of homogeneous quadratic optimization: approximation bounds and applications" Convex optimization in signal processing and communications, D.P. Palomar and Y.C. Eldar Eds., Cambridge University Press, 2009, ch. 4.

[11] S. Boyd and L. Vandenberghe, "Convex Optimization," Cambridge University Press, 2004.

[12] H. Lebret and S. Boyd, "Antenna pattern synthesis via convex optimization,” IEEE Trans. Signal Processing, vol. 45, no. 3, pp. 526-531, March 1997.

[13] Gurobi Optimizer Version 3.0. Houston, Texas: Gurobi Optimization, Inc., April 2010.

[14] M. Grant and S. Boyd. CVX: Matlab software for disciplined convex programming, version 2.1. http://cvxr.com/cvx, March 2014.

[15] C.A. Balanis, Antenna Theory-Analysis and Design, 2nd ed. New York: Wiley, 1996. 